An Extension of Implicit Monte Carlo Diffusion: Multigroup and The Difference Formulation

M. A. Cleveland, N. Gentile, T. S. Palmer

May 6, 2010

Journal of Computational Physics 
This document was prepared as an account of work sponsored by an agency of the United States government. Neither the United States government nor Lawrence Livermore National Security, LLC, nor any of their employees makes any warranty, expressed or implied, or assumes any legal liability or responsibility for the accuracy, completeness, or usefulness of any information, apparatus, product, or process disclosed, or represents that its use would not infringe privately owned rights. Reference herein to any specific commercial product, process, or service by trade name, trademark, manufacturer, or otherwise does not necessarily constitute or imply its endorsement, recommendation, or favoring by the United States government or Lawrence Livermore National Security, LLC. The views and opinions of authors expressed herein do not necessarily state or reflect those of the United States government or Lawrence Livermore National Security, LLC, and shall not be used for advertising or product endorsement purposes. 


\title{
An Extension of Implicit Monte Carlo Diffusion: Multigroup and The Difference Formulation
}

\author{
Mathew A. Cleveland ${ }^{\mathrm{a}, 1}$, Nick A. Gentile ${ }^{\mathrm{b}}$, Todd S. Palmer ${ }^{\mathrm{a}}$ \\ ${ }^{a}$ Oregon State University, Radiation Center, Corvallis, Oregon 97331 \\ ${ }^{b}$ Lawrence Livermore National Laboratory, Livermore, California 94550
}

\begin{abstract}
Implicit Monte Carlo (IMC) and Implicit Monte Carlo Diffusion (IMD) are approaches to the numerical solution of the equations of radiative transfer. IMD was previously derived and numerically tested on grey, or frequency-integrated problems [1]. In this research, we extend Implicit Monte Carlo Diffusion (IMD) to account for frequency dependence, and we implement the difference formulation [2] as a source manipulation variance reduction technique. We derive the relevant probability distributions and present the frequency dependent IMD algorithm, with and without the difference formulation. The IMD code with and without the difference formulation was tested using both grey and frequency dependent benchmark problems. The Su and Olson semi-analytic Marshak wave benchmark was used to demonstrate the validity of the code for grey problems [3]. The Su and Olson semi-analytic picket fence benchmark was used for the frequency dependent problems [4]. The frequency dependent IMD algorithm reproduces the results of both $\mathrm{Su}$ and Olson benchmark problems. Frequency group ref nement studies indicate that the computational cost of ref ning the group structure is likely less than that of group ref nement in deterministic solutions of the radiation diffusion methods. Our results show that applying the difference formulation to the IMD algorithm can result in an overall increase in the f gure of merit for frequency dependent problems. However, the creation of negatively weighted particles from the difference formulation can cause signif cant numerical instabilities in regions of the problem with sharp spatial gradients in the solution. An adaptive implementation of the difference formulation may be necessary to focus its use in regions that are at or near thermal equilibrium.
\end{abstract}

Key words: IMD, Non-Grey, Multigroup, The Difference Formulation

${ }^{*}$ Corresponding auther. Tel + 1541990 2126; fax + 541737 0480; e-mail: clevelam@onid.oregonstate.edu Preprint submitted to Journal of Computational Physics 


\section{Introduction}

The Implicit Monte Carlo (IMC) method has been shown to produce stable and robust solutions of the equations of radiative transfer in thick diffuse systems, but its computational costs can be prohibitively large, particularly in regions dominated by "effective scattering". There have been a variety of methods developed to deal with these inefficiencies $[5,6,1,7,11]$.

Symbolic Implicit Monte Carlo (SIMC) attempts to improve the efficiency of IMC using a spontaneous emission source with symbolic weights. If an emission source term is approximated as piecewise constant in a spatial cell, and the actual source distribution has a strong dependence on space in the cell, particles will artif cially be redistributed (or teleported) from regions where the source is large to regions where it is small. This discretization artifact is known as teleportation error [9]. The original form of SIMC which relies on piecewise constant basis functions does not approach the diffusion limit and suffers from teleportation error problems [9]. A piecewise linear SIMC was shown to approach the diffusion limit and is much less affected by teleportation error $[8,20]$. Teleportation error also occurs in IMC for small time steps [10]. SIMC also requires the solution of a non-linear system of equations at the end of the Monte Carlo simulation to determine the true weights used to approximate the spontaneous emission source. This can be expensive if the matrix is large, which is the case for systems with many spatial cells, or if the matrix is dense, which is the case when the system has large time steps [6].

For many years, researchers have employed the diffusion approximation to obtain the solution of radiative transfer problems in thick highly "scattering" regions. It is well known that the solution of the transport equation satisf es a diffusion equation as the background medium becomes optically thick and highly "scattering". This essentially means that the boundary layers are no longer strongly coupled to the solution of the problem. Most problems in high energy density physics contain some regions that are optically thick and some that are optically thin. This motivates the development of diffusion methods that can be easily coupled to either IMC or SIMC.

The "diffuse random walk" was proposed and developed to deal with the thick regions in these problems. A standard IMC algorithm was modif ed to approximate multiple "scatters" with a single diffusion event in these regions. Though it did show a potential speed-up, its most signif cant drawback was that it did a poor job of accounting for the change in angle that occurs after many scattering events. This meant that a standard IMC random walk had to be performed in between all diffuse random walks [5].

The second proposed method involved coupling standard SIMC and a deterministic method that solves the diffusion equation in thick regions [7]. This method successfully reproduced grey and frequency dependent one-dimensional benchmark solutions. Similar to standard SIMC, it is still necessary to solve a system of equations after the Monte Carlo transport, and teleportation error still accumulates in transport regions. More recent research has extended SIMC to include frequency dependence in the hybrid diffusion transport method. The difference formulation, discussed in detail below, for SIMC was also developed and has been shown to reduce variance in thick problems [9].

The difference formulation is a source manipulation variance reduction technique. A reference Planckian is subtracted from the photon energy density in both the transport and material energy equations, creating a new "transformed photon energy density". The transport operator remains unchanged, but the solution and source functions are changed. Solving this transformed transport problem with Monte Carlo involves the same interaction probabilities as the original radiative transfer equation. If the reference Planckian is set equal to the Planckian of the current 
material state, computational cost is shifted to regions where the material and photon energy densities are out of equilibrium. The difference formulation has been explored primarily in SIMC and has been shown to signif cantly increase the f gure of merit compared to the standard solution $[13,9]$. It has also been shown to yield promising results when used with IMC [14], but has not been explored in conjunction with Implicit Monte Carlo Diffusion (IMD) [1] or Discrete Diffusion Monte Carlo (DDMC) [11].

IMD and DDMC are similar in that they both apply the diffusion approximation to the effective scattering radiative transport equation, in spatially discretized form, and solve the resulting linear system of equations via Monte Carlo. However, they are different in that IMD treats time discretely and DDMC treats time as a continuous variable. Both methods have, to this date, only been tested on grey (frequency independent) radiative transfer problems. Frequency dependent diffusion models will be necessary to couple these diffusion methods to transport methods because of the strong frequency-dependence of the material opacities. In addition, the equations have only been solved in 1D slab or spherical geometry, or 2D cylindrical geometry on orthogonal or AMR grids $[1,11]$.

In this paper, we describe an extension of IMD to solve frequency dependent radiative transfer problems, and investigate the use of the difference formulation in grey and frequency dependent IMD. This publication outlines recent results described by Cleveland [12]. Though we have chosen to work with the IMD method, our approach can also be easily applied to the DDMC method. 


\section{Frequency-Dependent Radiative Transfer}

The frequency-dependent thermal photon transport equation,

$$
\frac{1}{c} \frac{\partial I[\bar{r}, v, \bar{\Omega}, t]}{\partial t}+\bar{\Omega} \cdot \bar{\nabla} I[\bar{r}, v, \bar{\Omega}, t]=-\sigma[v] I[\bar{r}, v, \bar{\Omega}, t]+\sigma[v] B[v, T],
$$

describes the photon distribution in a physical system. The photon distribution is tightly coupled to the material energy balance, which is represented mathematically as,

$$
\rho \frac{d \epsilon}{d t}=\iint d v d \bar{\Omega} \sigma[v] I[\bar{r}, v, \bar{\Omega}, t]-\iint d v d \bar{\Omega} \sigma[v] B[v, T] .
$$

In Eqs. (1) and (2), $c$ denotes the speed of light $[\mathrm{cm} / \mathrm{sec}], I[\bar{r}, v, \bar{\Omega}, t]$ is photon intensity [ergs/(s$\mathrm{cm}^{2}$-steradians-Hz)], $\bar{r}$ denotes a location in space [cm], $v$ denotes the photon frequency [Hz], $\bar{\Omega}$ denotes the direction of travel, $d \Omega$ is a solid angle [steradians], $t$ denotes time [sec], $\sigma$ denotes the opacity $[1 / \mathrm{cm}], B[v, T]$ is the Planck function [ergs/(s- $\mathrm{cm}^{2}$-steradians-Hz)], $T$ is the temperature of the background medium $[\mathrm{K}], \rho$ is the material mass density $\left[\mathrm{g} / \mathrm{cm}^{3}\right]$ and $\epsilon$ is the material specif c energy [ergs $/ g$ ]. The quantities $\sigma, T$, and $\epsilon$ are all a function of space $(r)$. In the interest of brevity the spatial dependence of these variables along with the photon intensity $(I)$ is assumed, but omitted in the notation.

The Planck function (or Planckian),

$$
B[v, T]=\frac{2 h}{c^{2}} \frac{v^{3}}{\left(e^{\frac{h \nu}{k T}}-1\right)},
$$

describes the frequency distribution of the photons being emitted from a material at temperature $T$ [K]. In this function $h$ is Planck's constant [ergs-sec] and $k$ is Boltzmann's constant [ergs/K].

Explicit temporal discretization of Eqs. (1) and (2) can become unstable for systems that have strong absorption and reemission $[1,15]$. This led Fleck and Cummings to develop an effective scattering which makes the equation unconditionally stable [15]. The Fleck factor, which can be described as the probability that an absorbed photon will not be reemitted during the current time step, is def ned as [15]

$$
f[T]=\frac{1}{\left(1+\frac{\Delta t c \sigma_{p} 4 T^{3}}{\rho c_{v}}\right)} .
$$

Here $c_{v}$ is the specif $\mathrm{c}$ heat of the material. The effective absorption opacity and scattering opacity are represented by $\sigma_{a}[v]=\sigma[v] f[T]$ and $\sigma_{s}[v]=\sigma[v](1-f[T])$ respectively. The f nal time-discretized transport and energy balance equations, in their unconditionally stable forms, are written as

$$
\begin{gathered}
\frac{1}{c} \frac{\Delta[[v, \bar{\Omega}]}{\Delta t}+\bar{\Omega} \cdot \bar{\nabla} I^{n+1}[v, \bar{\Omega}]+I^{n+1}[v, \bar{\Omega}] \sigma[v] \\
=\left.\frac{1}{4 \pi} c b\left[v,\left.T\right|^{n}\right] \sigma_{a}[v] a T^{4}\right|^{n}+\frac{1}{4 \pi} \frac{\sigma[v] b\left[v, T n^{n}\right]}{\sigma_{p}\left[\left.T\right|^{n}\right]} \iint d v d \bar{\Omega} I^{n+1}[v, \bar{\Omega}] \sigma_{s}[v],
\end{gathered}
$$

and

$$
\rho \frac{\Delta \epsilon}{\Delta t}=\iint d v d \bar{\Omega} I^{n+1}[v, \bar{\Omega}] \sigma[v] f\left[\left.T\right|^{n}\right]-\left.c \sigma_{p}\left[\left.T\right|^{n}\right] f\left[\left.T\right|^{n}\right] a T^{4}\right|^{n},
$$

respectively. Here

$$
b\left[v,\left.T\right|^{n}\right]=\frac{h}{\left.k T\right|^{n}} \frac{15}{\pi^{4}} \frac{\left(\frac{h v}{\left.k T\right|^{n}}\right)^{3}}{\left(e^{\frac{h v}{k T^{n}}}-1\right)}
$$


is the normalized Planck function where $\left.T\right|^{n}$ is the material temperature at time step $n$, and

$$
a=\frac{8 \pi^{5} k^{4}}{15 c^{3} h^{3}}
$$

is the radiation constant.

The frequency dependent diffusion equation can be derived from the transport equation by multiplying Eqs. (5) and (6) by $\frac{1}{c}$ and integrating over the angular variable [1]:

$$
\begin{gathered}
\frac{1}{c} \frac{\Delta e[v]}{\Delta t}+\frac{1}{c} \bar{\nabla} \cdot F^{n+1}[v]+e[v]^{n+1} \sigma[v] \\
=\left.b\left[v,\left.T\right|^{n}\right] \sigma_{a}[v] a T^{4}\right|^{n}+\frac{\sigma[v] b\left[v,\left.T\right|^{n}\right]}{\sigma_{p}\left[\left.T\right|^{n}\right]} \int d v e^{n+1}[v] \sigma_{s}[v], \\
\frac{\rho}{c} \frac{\Delta \epsilon}{\Delta t}=\int d v e^{n+1}[v] \sigma[v] f\left[\left.T\right|^{n}\right]-\left.\sigma_{p}\left[\left.T\right|^{n}\right] f\left[\left.T\right|^{n}\right] a T^{4}\right|^{n} .
\end{gathered}
$$

From this point forward, all temperature dependent data will be assumed to be evaluated at the previous time step unless otherwise specif ed; for example, $\left.T\right|^{n}$ will be simply denoted as $T$.

A relationship between the fux and energy density (Fick's law) [1] can derived by assuming that the photon intensity is linearly anisotropic ( $P_{1}$ approximation) and that the temporal derivative of the fux is small [16]:

$$
F[v]=-c D[v] \bar{\nabla} e[v]
$$

where $D[v]$ is the diffusion coefficient, including effective scattering, for frequency $v$,

$$
D[v]=\frac{1}{3 \sigma[v]} .
$$

This diffusion coefficient is derived under the assumption that the system has isotropic scattering. Note that without a "f ux limiter" it is possible for this method to allow particles to transport faster than the speed of light. This is an unphysical behavior that can cause wave fronts to propagate too quickly in some problems. Flux limiters can be used to prevent this behavior by forcing the diffusion coefficient to approach the proper limit as the system becomes optically thin. 


\section{Probabilistic Interpretation of the frequency-dependent Diffusion Equation}

In this section, we will derive a Monte Carlo method for approximating the solution of a frequency-dependent diffusion equation, coupled thermally with matter. This derivation will be independent of the details of the discretization of the diffusion equation and the matter energy equation. In particular, it does not depend on a specif c coordinate system, the number of spatial dimensions, or the specif $\mathrm{c}$ discretization of the fux. It does assume that both the diffusion equation and matter energy equation are differenced in time via forward Euler.

We will begin by considering the diffusion equation for radiation energy density $e$ with frequency-dependent absorption and scattering opacity. The units of $e$ are energy/length ${ }^{3}$. We will assume that the equation is discretized in space, so that the equation is written in terms of $e[v]_{i}$, which is the radiation energy density for mesh element $i$ with frequency $v$. We do not assume that the mesh elements are of any particular kind (e.g.,zones or nodes) or even that the set of elements are all of the same type. Quantities centered at time $\mathrm{n}$ will be indicated with a superscript $\mathrm{n}$, with a similar notation for time $\mathrm{n}+1$ quantities.

With the stated assumptions, the time-differenced diffusion equation is [1]

$$
\begin{aligned}
\frac{e[v]_{i}^{n+1}-e[v]_{i}^{n}}{\Delta t}+\bar{\nabla} \cdot F[v]_{i}^{n+1} & =-\left(\sigma_{a}[v]_{i}+\sigma_{s}[v]_{i}\right) c e[v]_{i}^{n+1} \\
& +\left.P_{t h}[v] c \sigma_{P} a T^{4}\right|_{i} \\
& +P_{s}[v] \int_{0}^{\infty} c e\left[v^{\prime}\right]_{i}^{n+1} \sigma_{s}\left[v^{\prime}\right]_{i} \mathrm{~d} v^{\prime}
\end{aligned}
$$

Here, $\sigma_{P}$ is the Planck mean opacity, and $P_{t h}$ is the probability distribution function for frequency of thermal emitted photons:

$$
P_{t h}[v]=\frac{\sigma_{a}[v] B[v, T]}{\int_{0}^{\infty} \sigma_{a}\left[v^{\prime}\right] B[v, T]}=\frac{\sigma_{a}[v] b[v, T]}{\sigma_{P}} .
$$

$B[v, T]$ is the Planck function, and $b[v, T]$ is the normalized Planck function:

$$
b[v, T]=\frac{B[v, T]}{\int_{0}^{\infty} B[v, T]} .
$$

$P_{s}[v]$ is the probability distribution function for frequency of scattered photons.

$\nabla \cdot F[v]_{i}^{n+1}$ is some approximation of the divergence of the fux at frequency $v$ for element $i$. We will make the further assumption that this quantity is a linear function of the $n+1$-st time step radiation energy density in the elements:

$$
\bar{\nabla} \cdot F[v]_{i}^{n+1}=\sum_{j} \mathcal{F}[v]_{i j} e[v]_{j}^{n+1}
$$

This assumption is necessary to make Eq.13 a linear equation in $e[v]_{i}^{n+1}$. Although we are assuming that $\bar{\nabla} \cdot F[v]_{j}^{n+1}$ is linear in $e[v]_{j}^{n+1}$, the $\mathcal{F}[v]_{i j}$ could be nonlinear functions of $v$ or $e[v]_{j}^{n}$, as would be the case if a $\mathrm{f}$ ux limiter was employed.

The frequency dependence of quantities in the above equations has been indicated explicitly by using the notation $[v]$. Thus, Eq.13 could describe $e[v]_{i}$ as a continuous function of $v$. But the $[v]$ notation used in Eq.13 could just as well formally describe a multigroup problem, in 
which the notation would indicate that the quantities were group integrated. For example, with the def nition

$$
e[v] \equiv \int_{v_{g}}^{v_{g+1}} e\left[v^{\prime}\right] \mathrm{d} v^{\prime},
$$

Eq.13 would describe the time evolution of a f nite set of group-integrated energies rather than a continuous function of frequency. We will refer to this case as the multigroup case. In the multigroup case, integrals over $v$ with limits 0 and $\infty$ have integrands which are step functions, and become sums over the groups. For example,

$$
\int_{0}^{\infty} e[v] \mathrm{d} v^{\prime}=\sum_{g=0}^{G} e_{g}\left[v_{g+1}-v_{g}\right]
$$

where $e_{g}$ denotes the frequency averaged group energy density and $G$ the number of groups. We will continue to use the notation $[v]$ to indicate that the Monte Carlo process described below is applicable to both continuous frequency and multigroup problems.

With the def nition in Eq. 16, Eq. 13 becomes

$$
\begin{aligned}
\frac{e[v]_{i}^{n+1}-e[v]_{i}^{n}}{\Delta t}+\sum_{j} \mathcal{F}[v]_{i j} e[v]_{j}^{n+1} & =-\left(\sigma_{a}[v]_{i}+\sigma_{s}[v]_{i}\right) c e[v]_{i}^{n+1} \\
& +\left.P_{t h}[v]_{i} c \sigma_{P} a T^{4}\right|_{i} \\
& +P_{s}[v]_{i} \int_{0}^{\infty} c e\left[v^{\prime}\right]_{i}^{n+1} \sigma_{s}\left[v^{\prime}\right]_{i} \mathrm{~d} v^{\prime}
\end{aligned}
$$

The discretized diffusion equation def nes a set (one for each value of $v$ ) of linear equations for the $e[v]_{j}^{n+1}$. Each of these linear equations can be written

$$
\sum_{j} \mathcal{A}[v]_{i j} e[v]_{j}^{n+1}=q[v]_{i}
$$

Here $q[v]_{i}$ is the sum of the thermal source term, the scattering source, and the radiation energy density at the old timestep:

$$
q[v]_{i}=P_{t h}[v]_{i} \sigma_{P} a T^{4}{ }_{i} c \Delta t+P_{s}[v]_{i} c \Delta t \int_{0}^{\infty} e\left[v^{\prime}\right]_{i}^{n+1} \sigma_{s}\left[v^{\prime}\right]_{i} \mathrm{~d} v^{\prime}+e[v]_{i}^{n}
$$

The diagonal of the matrix $\mathcal{A}[v]_{i j}$ has the value

$$
\mathcal{D}[v]_{j} \equiv \mathcal{A}[v]_{j j}=1+\Delta t \mathcal{F}[v]_{j j}+c \Delta t\left(\sigma_{a}[v]_{j}+\sigma_{s}[v]_{j}\right)
$$

and the off-diagonal, or fringe, terms are

$$
\mathcal{A}[v]_{i j}=\Delta t \mathcal{F}[v]_{i j}, i \neq j
$$

Linear systems such as Eq. 20 can be solved by many different means [17]. Since the scattering integral over $v$ couples the linear systems for all values of $v$ together, an iterative approach is often required [18]. 
The equation for $e[v]_{i}^{n+1}$ is accompanied by an equation for the matter specif c energy, $\epsilon$, whose units are energy/mass:

$$
\rho \frac{\mathrm{d} \epsilon}{\mathrm{d} t}=\int_{0}^{\infty} c \sigma_{a}\left[v^{\prime}\right] e\left[v^{\prime}\right]-c \sigma_{P} a T^{4}
$$

(In this equation, and the rest of this work, we have assumed that the mass density $\rho$ is constant in time.) In both Eq.19 and Eq. 24, we have left the time-centering of the matter temperature $T$ unspecif ed. Various solution techniques may set specif $\mathrm{c}$ values for the time-centering, or use various approximations. The Monte Carlo process we will describe below is independent of the specif c time centering assumed for $T$ in the thermal source term.

The time discretization of Eq. 24 via forward Euler results in

$$
\rho \frac{\epsilon_{i}^{n+1}-\epsilon_{i}^{n}}{\Delta t}=\int_{0}^{\infty} c \sigma_{a}\left[v^{\prime}\right]_{i} e\left[v^{\prime}\right]_{i}^{n+1}-\left.c \sigma_{P} a T^{4}\right|_{i}
$$

The integral over $v$, containing $e\left[v^{\prime}\right]_{i}^{n+1}$, is a forward time-centered approximation for energy absorbed by the matter from the radiation.

We will now present Monte Carlo estimates for the quantities $e_{i}^{n+1}$ in equation Eq.19 and $e$ in Eq. 24. Our development of the Monte Carlo solution will solve Eq.19 by moving particles among the elements $i$ on which $e$ is def ned. The particles will be born in elements, move among them, and quantities will be tallied in the elements with certain probabilities to be presented below. After these probabilities are def ned, we will show that the expectation value of one tallied quantity, in the limit of an inf nite number of particles, will yield a value for $e[v]_{i}^{n+1}$ that satisf es Eq.19. Another tallied quantity will, in the same limit, yield a value for $e$ that satisf es Eq.24.

Since the quantities we calculate satisfy Eq.19 and Eq.24 exactly in the limit of an inf nite number of particles, we will take the estimates $e[v]_{i}^{n+1}$ and $e$ obtained from simulations using a $\mathrm{f}$ nite number of particles as Monte Carlo solutions of these equations.

The Monte Carlo solution process will involve $N$ particles. These particles will have a total energy of

$$
E_{\text {total }} \equiv \sum_{i} \int_{0}^{\infty} e[v]_{i}^{n} \mathrm{~d} v V_{i}+c \sigma_{P} a T^{4}{ }_{i} V_{i} \Delta t .
$$

Here, $V_{i}$ is the volume associated with element $i . E_{\text {total }}$ is the total radiation energy in the problem at $t_{n}$. (If there were additional sources of radiation energy in the problem, those would be added to Eq.26.) Each particle will carry an energy $E_{p} \equiv E_{\text {total }} / N$.

The number of particles with frequency $v$ to be created in element $i$ will be

$$
N^{c}[v]_{i} \equiv N \frac{e[v]_{i}^{n} V_{i}+\left.P_{t h}[v] c \sigma_{P} a T^{4}\right|_{i} V_{i} \Delta t}{E_{\text {total }}} .
$$

Subsequent to its creation, each particle will undergo the following events, with the stated probabilities:

- a particle may move from element $i$ to a different element $j$ with probability

$$
\mathcal{P}^{m}[v]_{i j} \equiv \frac{-\Delta t \mathcal{F}[v]_{j i}}{\mathcal{D}[v]_{i}}
$$


Note that particles only move between elements that contribute non-zero values to the discretization of the $\operatorname{fux} \mathcal{F}$;

- a particle in element $i$ may scatter, changing its frequency, with probability

$$
\mathcal{P}^{s}[v]_{i} \equiv \frac{c \Delta t \sigma_{s}[v]_{i}}{\mathcal{D}[v]_{i}}
$$

upon scattering, the new frequency of the particle will be drawn from the probability distribution function $\left.P_{S}[v]\right]_{i}$;

- a particle in element $i$ may be absorbed with probability

$$
\mathcal{P}^{a}[v]_{i} \equiv \frac{c \Delta t \sigma_{a}[v]_{i}}{\mathcal{D}[v]_{i}}
$$

upon absorption, the particle is terminated and its energy $E_{p}$ is tallied into quantity $E^{a}[v]_{i}$;

- a particle in element $i$ may reach census with probability

$$
\mathcal{P}^{c}[v]_{i} \equiv \frac{1}{\mathcal{D}[v]_{i}}
$$

upon reaching census, the particle is advanced no further, and its energy $E_{p}$ is tallied into quantity $E^{c}[v]_{i}$.

The Monte Carlo process described above will result in values $E^{c}[v]_{i}$ and $E_{i}^{a}$ for every element. We will now show that these quantities, in the limit $N \rightarrow \infty$, will produce estimates for $e[v]_{i}^{n+1}$ and $\int c \sigma_{a}\left[v^{\prime}\right]_{i} e[v]_{i}^{n+1} d v^{\prime}$ which satisfy Eq. 19 and Eq. 25 respectively.

We begin by considering the quantity $N[v]_{i}$, which we def ne as the number of particles with frequency $v$ which pass through element $i$ during a given time step (that is, in the time $\left.\left[t^{n}, t^{n+1}\right].\right) N[v]_{i}$ will equal the sum of the number of particles created in element $i$ originally, plus the number which move into the element from neighboring elements, plus the number already in the element, but with a different frequency $v^{\prime}$, which scatter into the frequency $v$.

In the limit $N \rightarrow \infty$, we can express each of the quantities that contribute to $N[v]_{i}$ in terms of the probabilities def ned above multiplied by the number of particles that have passed through neighboring elements, $N[v]_{j}$. For example, we can express the number of particles which move into the element $i$ from neighboring elements as $\sum_{j \neq i} \mathcal{P}^{m}[v]_{j i} N[v]_{j}$.

Thus, as $N \rightarrow \infty, N[v]_{i}$ will satisfy

$$
N[v]_{i}=N^{c}[v]_{i}+\sum_{j \neq i} \mathcal{P}^{m}[v]_{j i} N[v]_{j}+P_{s}[v]_{i} \int_{0}^{\infty} \mathcal{P}^{s}\left[v^{\prime}\right]_{i} N\left[v^{\prime}\right]_{i} \mathrm{~d} v^{\prime}
$$

Next, we will use the number balance equation, Eq. 32, to derive an expression for $E^{c}[v]_{i}$, the energy that reaches census with frequency $v$. The value of $E^{c}[v]_{i}$ will be the census probability multiplied by the energy of every particle that has ever entered the element:

$$
\begin{aligned}
E^{c}[v]_{i} & =\mathcal{P}^{c}[v]_{i} E_{p} N[v]_{i} \\
& =\mathcal{P}^{c}[v]_{i} E_{p} N^{c}[v]_{i} \\
& +\mathcal{P}^{c}[v]_{i} \sum_{j \neq i} \mathcal{P}^{m}[v]_{j i} E_{p} N[v]_{j} \\
& +\mathcal{P}^{c}[v]_{i} P_{s}[v]_{i} \int_{9}^{\infty} \mathcal{P}^{s}\left[v^{\prime}\right]_{i} E_{p} N\left[v^{\prime}\right]_{i} \mathrm{~d} v^{\prime}
\end{aligned}
$$


The frst term in Eq. 33 can be rewritten by using Eqs. 27 and 31 and the def nition of $E_{p}$ :

$$
\mathcal{P}^{c}[v]_{i} E_{p} N[v]_{i}=\frac{e[v]_{i}^{n} V_{i}+\left.P_{t h}[v]_{i} c \sigma_{P} a T^{4}\right|_{i} V_{i} \Delta t}{\mathcal{D}[v]_{i}}
$$

The second term, using Eq. 28 inside the sum, can be rewritten

$$
\begin{aligned}
\mathcal{P}^{c}[v]_{i} \sum_{j \neq i} \mathcal{P}^{m}[v]_{j i} E_{p} N[v]_{j} & =-\sum_{i \neq j} \frac{1}{\mathcal{D}[v]_{i}} \frac{\Delta t \mathcal{F}[v]_{i j}}{\mathcal{D}[v]_{j}} E_{p} N[v]_{j} \\
& =-\sum_{i \neq j} \Delta t \mathcal{F}[v]_{i j} E^{c}[v]_{j} \frac{1}{\mathcal{D}[v]_{i}}
\end{aligned}
$$

The last term, using using Eq. 29, can be rewritten

$$
\mathcal{P}^{c}[v]_{i} P_{s}[v]_{i} \int_{0}^{\infty} \mathcal{P}^{s}\left[v^{\prime}\right]_{i} E_{p} N\left[v^{\prime}\right]_{i} \mathrm{~d} v^{\prime}=\frac{P_{s}[v]_{i}}{\mathcal{D}[v]_{i}} \int_{0}^{\infty} c \Delta t \sigma_{s}\left[v^{\prime}\right]_{i} E^{c}\left[v^{\prime}\right]_{i} \mathrm{~d} v^{\prime}
$$

With these changes, Eq. 33 becomes

$$
\begin{aligned}
E^{c}[v]_{i} & =\frac{e[v]_{i}^{n} V_{i}+\left.P_{t h}[v]_{i} c \sigma_{P} a T^{4}\right|_{i} V_{i} \Delta t}{\mathcal{D}[v]_{i}} \\
& -\sum_{i \neq j} \Delta t \mathcal{F}[v]_{i j} E^{c}[v]_{j} \frac{1}{\mathcal{D}[v]_{i}} \\
& +\frac{P_{s}[v]_{i}}{\mathcal{D}[v]_{i}} \int_{0}^{\infty} c \Delta t \sigma_{s}\left[v^{\prime}\right]_{i} E^{c}\left[v^{\prime}\right]_{i} \mathrm{~d} v^{\prime}
\end{aligned}
$$

Multiplying through by $\mathcal{D}[v]_{i} / \Delta t$ yields

$$
\begin{aligned}
\frac{\mathcal{D}[v]_{i}}{\Delta t} E^{c}[v]_{i} & =\frac{1}{\Delta t} e[v]_{i}^{n} V_{i}+\left.P_{t h}[v]_{i} \sigma_{P} a T^{4}\right|_{i} V_{i} \\
& -\sum_{i \neq j} \mathcal{F}[v]_{i j} E^{c}[v]_{j} \\
& +P_{s}[v]_{i} \int_{0}^{\infty} c \sigma_{s}\left[v^{\prime}\right]_{i} E^{c}\left[v^{\prime}\right]_{i} \mathrm{~d} v^{\prime}
\end{aligned}
$$

Using the def nition of $\mathcal{D}[v]_{i}$, Eq. 22, and collecting terms in $\mathcal{F}_{i j}$, we obtain

$$
\begin{aligned}
\frac{E^{c}[v]_{i}-e[v]_{i}^{n} V_{i}}{\Delta t}+\sum_{j} \mathcal{F}[v]_{i j} E^{c}[v]_{j} & =-c\left(\sigma_{a}[v]_{i}+\sigma_{s}[v]_{i}\right) E^{c}[v]_{i} \\
& +P_{t h}[v]_{i} c \sigma_{P} a T^{4}{ }_{i} V_{i} \\
& +P_{s}[v]_{i} \int_{0}^{\infty} c \sigma_{s}\left[v^{\prime}\right]_{i} E^{c}\left[v^{\prime}\right]_{i} \mathrm{~d} v^{\prime}
\end{aligned}
$$

Finally, dividing by $V_{i}$ and def ning $e^{c}[v]_{i} \equiv E^{c}[v]_{i} / V_{i}$, we obtain

$$
\begin{aligned}
\frac{e^{c}[v]_{i}-e[v]_{i}^{n}}{\Delta t}+\sum_{j} \mathcal{F}[v]_{i j} e^{c}[v]_{j} & =-c\left(\sigma_{a}[v]_{i}+\sigma_{s}[v]_{i}\right) e^{c}[v]_{i} \\
& +\left.P_{t h}[v]_{i} c \sigma_{P} a T^{4}\right|_{i} \\
& +P_{s}[v]_{i} \int_{0}^{\infty} c \sigma_{s}\left[v^{\prime}\right]_{i} e^{c}\left[v^{\prime}\right]_{i} \mathrm{~d} v^{\prime} \\
10 &
\end{aligned}
$$


By comparing Eq. 40 to Eq. 19, we see that $e^{c}[v]_{i}$ satisf es the diffusion equation. Since this quantity, which is calculated in the limit of an inf nite number of particles, is a solution of the equation we are trying to solve, we can take the value of $e_{i}^{c}$ obtained with a f nite number of particles as a Monte Carlo estimate of the solution of the diffusion equation, $e^{n+1}[v]_{i}$.

We will now examine $E^{a}[v]_{i}$, the other quantity tallied in the Monte Carlo process. In the limit of an inf nite number of particles, we have

$$
E^{a}[v]_{i}=\mathcal{P}^{a}[v]_{i} E_{p} N[v]_{i}
$$

Using the def nitions of $\mathcal{P}^{a}[v]_{i}$, Eq. 30, $\mathcal{P}^{c}[v]_{i}$, Eq. 31, and the fact that $E^{c}[v]=\mathcal{P}^{c}[v] E_{p} N[v]$, we obtain

$$
\begin{aligned}
E^{a}[v]_{i} & =\frac{c \Delta t \sigma_{a}[v]_{i}}{\mathcal{D}[v]_{i}} E_{p} N[v] \\
& =c \Delta t \sigma_{a}[v]_{i} E_{p} N[v] P^{c}[v]_{i} \\
& =c \Delta t \sigma_{a}[v]_{i} E^{c}[v]_{i}
\end{aligned}
$$

Dividing by the element volume $V_{i}$ and integrating over the frequency yields

$$
e_{i}^{a}=\int_{0}^{\infty} c \sigma_{a}[v]_{i} \Delta t e^{c}[v]_{i} \mathrm{~d} v
$$

Since $e^{c}[v]_{i}$ has been shown to be an estimate for $e[v]_{i}^{n+1}, e_{i}^{a}$ is an estimate for the integral in Eq. 43. (For a f nite number of particles, $e_{i}^{a}$ would be formed by summing Eq. 41 over groups in each zone and dividing by the zone volume.) Thus $e_{i}^{a}$ can be inserted into the discretized matter energy equation, Eq. 25, in place of the integral over $e[v]_{i}^{n+1}$, which describes the energy absorbed by the matter from the radiation. This allows us to solve

$$
\rho\left(\epsilon_{i}^{n+1}-\epsilon_{i}^{n}\right)=e_{i}^{a}-\left.c \sigma_{P} a T^{4}\right|_{i} \Delta t
$$

for the time $n+1$ matter specif c energy $\epsilon_{i}^{n+1}$.

We have now demonstrated that solutions for both Eq. 19 and Eq. 25 are provided by the Monte Carlo procedure outlined above.

The probabilities used in this Monte Carlo process can be expressed in terms of the components of the matrix $\mathrm{A}$ in the standard linear system version of the diffusion equation, Eq. 20. Using the def nitions of the probabilities in Eqs. 28 through 31, and the equations describing the linear system, Eqs. 20 through 23, we f nd that the probability for a particle to move from element $i$ to element $j$ is

$$
\mathcal{P}^{m}[v]_{i j}=-\mathcal{A}[v]_{j i} / \mathcal{A}[v]_{i i} .
$$

The probability that a particle may scatter is

$$
\mathcal{P}^{s}[v]_{i} \equiv c \Delta t \sigma_{s}[v]_{i} / \mathcal{A}[v]_{i i} .
$$

In the event of a scatter, a new frequency is selected from the probability distribution function $P_{s}[v]$.

The probability for particle to be absorbed is

$$
\mathcal{P}^{a}[v]_{i} \equiv c \Delta t \sigma_{a}[v]_{i} / \mathcal{A}[v]_{i i}
$$


Finally, the probability for a particle to reach census is

$$
\mathcal{P}^{c}[v]_{i} \equiv 1 / \mathcal{A}[v]_{i i} .
$$

Any discretization of Eq.13 that results in a linear system can be solved via a Monte Carlo method as well as by the usual techniques that involve linear system solutions, by deriving the necessary probabilities from the matrix $\mathcal{A}_{i j}$.

In the Monte Carlo algorithm, the scattering is handled by changing the frequency of the particles. This couples all the groups together during the advance of the particles, so there is no need to iterate over $v$.

We have referred to the quantities def ned by Eqs. 45 - 48 as probabilities, but have not shown that they are, in general, positive and add to one. In general, they do not. Whether they do satisfy these conditions depends on the discretization of the $\mathrm{f} u x$. The sum of the probabilities is, from Eqs. 45 - 48,

$$
\frac{1+c\left(\sigma_{a}[v]_{i}+\sigma_{s}[v]_{i}\right) \Delta t-\sum_{j \neq i} \mathcal{A}[v]_{j i}}{\mathcal{D}[v]_{i}} .
$$

This will sum to 1 if the numerator equals the denominator. Using Eq. 22, this holds if

$$
-\sum_{j \neq i} \mathcal{A}[v]_{j i}=\mathcal{A}[v]_{i i},
$$

which implies

$$
\sum_{j} \mathcal{A}[v]_{j i}=0 .
$$

Eq. 51 is satisf ed by most of the discretization schemes of which the authors are aware.

The probabilities given by Eq. 46 and Eq. 47 are manifestly positive. The probability given by Eq. 48 will be positive if the diagonal elements of the matrix are positive. The probability given by Eq. 45 will be positive if the fringes of the matrix $\mathcal{A}[v]$ are negative. This is the case for most $\mathrm{f}$ nite difference and $\mathrm{f}$ nite element discretization schemes on orthogonal grids. On nonorthogonal grids, Eq. 45 can give a negative probability of moving from element $i$ to element $j$.

In this paper, we will examine only one-dimensional Cartesian discretization schemes which satisfy both conditions on the probabilities. In a future work, we will examine more complicated discretization schemes, and look at Monte Carlo methods that result when one or both conditions is/are not met.

\section{Implicit Monte Carlo Diffusion Probabilities for 1-D Slab Geometry}

We now introduce the spatial discretization of the 1-D slab geometry diffusion equation. The specif $\mathrm{c}$ form of the matrix for this cell-centered discretization uniquely def nes the probabilities in the Monte Carlo process via Eqs. (45) through (48). We employ the cell-centered discretization of Pomraning and Szilard [19] which yields a tridiagonal system of equations for the cell-centered energy density.

We will employ the technique of Fleck and Cummings [15] in the diffusion equation, as described in [1], to enhance stability. This technique reduces the absorption opacity by a factor of $f$ 
def ned in Eq. 4, and introduces an effective scattering of $(1-f) \sigma_{a}$. Note that the effective scattering has a probability distribution function of $\frac{\sigma_{a}[v] b[v, T]}{\sigma_{p}}$ while the physical scattering described by $\sigma_{s}[v]$ will in general have a probability distribution function $P_{s}[v]$.

This cell centered discretization can be written in terms of the coefficient matrix terms ( $\mathcal{A}[v]$, $\mathcal{D}[v])$ def ned in Eqs. 22 and 23:

$$
\mathcal{A}[v]_{j, j-1} e[v]_{j-1}^{n+1}+\mathcal{D}[v]_{j} e[v]_{j}^{n+1}+\mathcal{A}[v]_{j, j+1} e[v]_{j+1}^{n+1}=q[v]_{j},
$$

where

$$
\begin{gathered}
\mathcal{A}[v]_{j, j-1}=-c \frac{2 \Delta t D[v]_{j-1} D[v]_{j}}{\Delta x_{j}\left(\Delta x_{j} D[v]_{j-1}+\Delta x_{j-1} D[v]_{j}\right)}, \\
\mathcal{A}[v]_{j, j+1}=-c \frac{2 \Delta t D[v]_{j} D[v]_{j+1}}{\Delta x_{j}\left(\Delta x_{j+1} D[v]_{j}+\Delta x_{j} D[v]_{j+1}\right)}, \\
\mathcal{D}[v]_{j}=1+c \Delta t f[T] \sigma_{a}[v]+c \Delta t(1-f[T]) \sigma_{a}[v]+c \Delta t \sigma_{s}[v]-\mathcal{A}[v]_{j, j-1}-\mathcal{A}[v]_{j, j+1},
\end{gathered}
$$

and

$$
\begin{aligned}
q[v]_{j}=\left.c \Delta t f[T] \sigma_{p}[T] a T^{4}\right|_{j} P_{t h}[v] & +c \Delta t \frac{\sigma_{a}[v] b[v, T]}{\sigma_{p}} \int d v e[v]_{j}^{n+1}(1-f[T]) \sigma_{a}[v] \\
& +c \Delta t P_{s}[v] \int d v e[v]_{j}^{n+1} \sigma_{s}[v] \\
& +e[v]_{j}^{n} .
\end{aligned}
$$

In Eqs. 53, 54, and 55, $D[v]_{j}$ is the diffusion coefficient, including effective scattering, in cell $j$ for frequency $v, \Delta t$ is the current time step size, and $\Delta x_{j}$ is the size of cell $j$. 


\section{The Difference Formulation}

The difference formulation is a variance reduction technique which changes the source of the problem to focus the computational work where the solution is rapidly changing [9]. We def ne a new quantity, $e_{d}[v]$, which is the difference between the radiation energy density and the Planckian def ned at some user-specif ed temperature $T_{d}$,

$$
e_{d}[v]=e[v]-a T_{d}^{4} b\left[v, T_{d}\right]
$$

$T_{d}$ can be any temperature, but the stability of the method is heavily dependent on this choice [13]. This is a potentially useful approach because if the radiation is near equilibrium with the matter at temperature $T_{d}$, then $e_{d}$ will be small.

If we replace $e$ with $e_{d}+a T_{d}^{4} b$ in the diffusion equation, we obtain a diffusion equation for $e_{d}$ :

$$
\begin{gathered}
\frac{1}{c} \frac{\Delta e_{d}[v]}{\Delta t}+\frac{1}{c} \bar{\nabla} \cdot F_{d}[v]^{n+1}=-e_{d}[v]^{n+1} \sigma[v] \\
+b[v, T] \sigma[v] f[T] a T^{4}+\frac{\sigma[v] b[v, T]}{\sigma_{p}} \int \mathrm{d} v e_{d}[v]^{n+1} \sigma[v](1-f[T]) \\
-b\left[v, T_{d}\right] a T_{d}^{4} \sigma[v]+\frac{\sigma[v] b[v, T]}{\sigma_{p}} \int \mathrm{d} v b\left[v, T_{d}\right] a T_{d}^{4} \sigma[v](1-f[T]) \\
-\frac{1}{c} \frac{\Delta b\left[v, T_{d}\right] a T_{d}^{4}}{\Delta t}-\bar{\nabla}\left(-D[v] \bar{\nabla} b\left[v, T_{d}\right] a T_{d}^{4}\right) .
\end{gathered}
$$

If the new scattering term containing the normalized Planckian evaluated at the "reference" temperature in Eq. (58) is integrated and expanded, the new form of the transformed diffusion equation is

$$
\begin{gathered}
\frac{1}{c} \frac{\Delta e_{d}[v]}{\Delta t}+\frac{1}{c} \bar{\nabla} \cdot F_{d}[v]^{n+1}=-e_{d}[v]^{n+1} \sigma[v] \\
+\frac{\sigma[v] b[v, T]}{\sigma_{p}} \int \mathrm{d} v e_{d}[v]^{n+1} \sigma[v](1-f[T]) \\
+b[v, T] \sigma[v] f[T] a\left(T^{4}-T_{d}^{4} \frac{\sigma_{d_{p}}}{\sigma_{p}}\right) \\
+a T_{d}^{4}\left(\frac{\sigma_{d_{p}}}{\sigma_{p}} b[v, T] \sigma[v]-b\left[v, T_{d}\right] \sigma[v]\right) \\
-\frac{1}{c} \frac{\Delta b\left[v, T_{d}\right] a T_{d}^{4}}{\Delta t}-\bar{\nabla}\left(-D[v] \bar{\nabla} b\left[v, T_{d}\right] a T_{d}^{4}\right),
\end{gathered}
$$

where $\sigma_{d_{p}}$ is the Planck opacity evaluated at the reference temperature. If the reference temperature is chosen to be piecewise constant, the time derivative of the transformed source term is zero. However, the reference temperature is dependent on space and using the second order differencing of the spatial gradient, we fnd

$$
\begin{gathered}
\bar{\nabla}\left(-D[v] \bar{\nabla} b\left[v, T_{d}\right] a T_{d}^{4}\right)= \\
-c \frac{2 D[v]_{j} D[v]_{j+1}}{\Delta x_{j}\left(\Delta x_{j+1} D[v]_{j}+\Delta x_{j} D[v]_{j+1}\right)}\left(\left.b\left[v,\left.T_{d}\right|_{j+1}\right] a T_{d}^{4}\right|_{j+1}-\left.b\left[v,\left.T_{d}\right|_{j}\right] a T_{d}^{4}\right|_{j}\right) \\
+c \frac{2 D[v]_{j-1} D[v]_{j}}{\Delta x_{j}\left(\Delta x_{j} D[v]_{j-1}+\Delta x_{j-1} D[v]_{j}\right)}\left(\left.b\left[v,\left.T_{d}\right|_{j}\right] a T_{d}^{4}\right|_{j}-\left.b\left[v,\left.T_{d}\right|_{j-1}\right] a T_{d}^{4}\right|_{j-1}\right) .
\end{gathered}
$$

It is possible to combine Eqs. (59) and (60) to yield a familiar form of the diffusion equation (Eq. 9).

$$
\begin{gathered}
\frac{1}{c} \frac{\Delta e_{d}[v]}{\Delta t}+\frac{1}{c} \bar{\nabla} \cdot F_{d}[v]^{n+1}+e_{d}[v]^{n+1} \sigma[v]= \\
+\frac{\sigma[v] b[v, T]}{\sigma_{p}} \int \mathrm{d} v e_{d}[v]^{n+1} \sigma[v](1-f[T])-\bar{\nabla}\left(-D[v] \bar{\nabla} b\left[v, T_{d}\right] a T_{d}^{4}\right) . \\
14
\end{gathered}
$$


In fact, this equation is identical to Eq. (9) with a new source term replacing the thermal emission source, provided the reference temperature is constant over a single time step and equal to the local material temperature.

The coefficient matrix for the solution of the discretized difference formulation equations is the same as that of the standard diffusion equation. This means all the probabilities that were developed previously are applicable to difference formulation Monte Carlo simulations. The result of the simulations, however, is "transformed" energy density $\left(e_{d}[v]^{n+1}\right)$ rather than the actual energy density $\left(e[v]^{n+1}\right)$. After the "transformed" energy density is found for a given time step, it is possible to determine the actual energy density by adding it to $a T_{d}^{4} b\left[v, T_{d}\right]$.

The difference formulation also changes the material energy balance equation. The absorption tally is identical in the transformed system to that of the standard system. However, the census tally involves $e_{d}[v]$ rather than $e[v]$. In the difference formulation, the energy absorbed into the material $\left(e_{d, i}^{a}\right)$ can be written as:

$$
e_{d, i}^{a}=\int \mathrm{d} v c \Delta t \sigma[v] f\left[\left.T\right|_{i}\right] e_{d}[v]_{i}
$$

Using Eq. (57) and integrating simplif es the expression to the Planck distribution,

$$
e_{d, i}^{a}=\int \mathrm{d} v c \Delta t \sigma[v] f\left[\left.T\right|_{i}\right] e[v]_{i}-\left.c \Delta t \sigma_{p} f\left[\left.T\right|_{i}\right] a T_{d}^{4}\right|_{i}
$$

This means that the absorption tally of $e_{d}[v]$ is equal to the change in the material energy density if the reference temperature is equal to the material temperature:

$$
\rho \Delta \epsilon=e_{d}^{a} .
$$

The derivation of the difference formulation is straightforward; creating the appropriate frequency distribution for sampling is more complex. In the standard Monte Carlo case, the frequency distribution is described by a single Planckian, Eq. (3), and the current census distribution. When the difference formulation is used, there are a number of new source distributions that must be sampled. In the standard form, the initial census distribution carries over from the census distribution at the end of the last time step. In the difference formulation, the Planck function at the reference temperature $T_{d}$ is subtracted from the initial photon density (Eq. 57) changing the magnitude of the initial transformed photon density and its frequency distribution. This means that the energy needs to be added either into the photon energy density using appropriate frequency bins (for multigroup methods), or as particles (for continuous or multigroup frequencies) with the following probability distribution function $P D F^{b}[v]$;

$$
P D F^{b}[v]=\frac{b\left[v, T_{d}\right]}{\int_{0}^{\infty} \mathrm{d} v b\left[v, T_{d}\right]}=b\left[v, T_{d}\right] .
$$

In the grey case, this distribution is irrelevant and the transformed census energy can just be subtracted from the total census. In the multigroup or continuous energy case, if the subtracted reference energy is added to the census using a distribution of negatively weighted particles, it may increase the variance rather than decreasing it. The census distribution will no longer be positive; to avoid this, it is possible to create a grouped frequency distribution function that spans the range of frequencies currently represented in census. The transformed census for each group becomes

$$
e_{d}\left[v^{k}\right]=e\left[v^{k}\right]-a T_{d}^{4} \int_{v_{k-1}}^{v_{k}} \mathrm{~d} v P D F^{b}[v]=e\left[v^{k}\right]-a T_{d}^{4} \int_{v_{k-1}}^{v_{k}} \mathrm{~d} v b\left[v, T_{d}\right] .
$$


After the initial photon density has been modif ed, it is necessary to determine the frequency distribution of the new spatial derivative in the transformed diffusion equation (Eq. (60)). This streaming source term is treated as two separate sources, each with different opacity-dependent coefficients. These two sources can be def ned as:

$$
q_{d}^{-}[v]_{j}=-c \frac{2 D[v]_{j-1} D[v]_{j}}{\Delta x_{j}\left(\Delta x_{j} D[v]_{j-1}+\Delta x_{j-1} D[v]_{j}\right)}\left(\left.b\left[v,\left.T_{d}\right|_{j-1}\right] a T_{d}^{4}\right|_{j-1}-\left.b\left[v,\left.T_{d}\right|_{j}\right] a T_{d}^{4}\right|_{j}\right)
$$

and

$$
q_{d}^{+}[v]_{j+1}=c \frac{2 D[v]_{j} D[v]_{j+1}}{\Delta x_{j}\left(\Delta x_{j+1} D[v]_{j}+\Delta x_{j} D[v]_{j}\right)}\left(\left.b\left[v,\left.T_{d}\right|_{j+1}\right] a T_{d}^{4}\right|_{j+1}-\left.b\left[v,\left.T_{d}\right|_{j}\right] a T_{d}^{4}\right|_{j}\right) .
$$

These two source terms both operate in zone $j$, and can have either sign. It is easily shown that $q_{j+1}^{-}=-q_{j}^{+}$, so the positive energy particles sourced into zone $j$ are cancelled by negative particles sourced into zones $j-1$ and $j+1$. The streaming source term adds no energy to the problem. It is analagous to the face source term that arises in the difference formulation of the transport equation [9], which gives rise to negative photons entering the hotter zone adjacent to a face, and an equal amount of positive energy photons entering the colder zone.

For multigroup methods, Eqs. (67) and (68) provide the probability distribution functions for the frequency of streaming particles:

$$
\operatorname{PDF}^{q_{d}}[v]_{j-1}=\frac{q_{d}[v]_{j-1}}{\int_{0}^{\infty} \mathrm{d} v q_{d}[v]_{j-1}}
$$

and

$$
P D F^{q_{d}}[v]_{j+1}=\frac{q_{d}[v]_{j+1}}{\int_{0}^{\infty} \mathrm{d} v q_{d}[v]_{j+1}} .
$$

These PDFs generate cumulative probability distribution functions (CDF) and an event type can be sampled using a pseudorandom number distributed uniformly between 0 and 1 . These CDFs are given by

$$
C D F^{q_{d}}\left[v_{k}\right]_{j-1}=\frac{\sum_{0}^{k} \int_{v_{k-1}}^{v_{k}} \mathrm{~d} v q_{d}[v]_{j-1}}{\int_{0}^{\infty} \mathrm{d} v q_{d}[v]_{j-1}}
$$

and

$$
C D F^{q_{d}}\left[v_{k}\right]_{j+1}=\frac{\sum_{0}^{k} \int_{v_{k-1}}^{v_{k}} \mathrm{~d} v q_{d}[v]_{j+1}}{\int_{0}^{\infty} \mathrm{d} v q_{d}[v]_{j+1}} .
$$

This method of distributing particles in frequency can be computationally expensive if performed for every frequency selection. Improved performance may be possible using tabulated functions or other selection techniques similar to those used in SIMC In Brooks' approach, the streaming operator is independent of opacity unlike the streaming source in our work. Tabulating the distribution functions is likely a more usable approach.

After the new transformed census value is determined, the amount of initial reference energy must be added back into the system to obtain the energy density:

$$
e[v]^{n+1}=e_{d}[v]_{16}^{n+1}+a T_{d}^{4} b\left[v, T_{d}\right] .
$$


Again, it is necessary to consider the frequency distribution (described by equation 65) when adding this energy back into the photon energy density solution. In this research, this addition is performed in the same fashion as the initial subtraction, with the exception of when no particles registered in census. In this case, particles were created with the frequency distribution of $b\left(v, T_{d}\right)$ and weights that sum to $a T_{d}^{4}$. The frequency distribution of the normalized Planckian for this work was selected using the efficient algorithm described by Barnett and Canf eld [21]. 


\section{Results}

We frst test the implementation of IMD on a one-dimensional grey test problem [1]. This problem consists of an initially cold semi-inf nite body that has a f ux incident on the left face. This problem has a normalized semi-analytic solution [3]. The Su and Olson results were translated into a normalized form to allow comparison with the IMD code results using the constants def ned in Table 1. The unit (Mm) denotes mega-meters (or 1e+6 meters). All other parameters used for the problems solved in this work are displayed in Table 2.

\begin{tabular}{|c||c|c|c|}
\hline \multicolumn{4}{|c|}{ Constants } \\
\hline Constant & Symbol & Value & Units \\
\hline \hline Planck & $h$ & $6.626 \mathrm{E}-34$ & joule*sec. \\
\hline Boltzmann & $k$ & $1.381 \mathrm{E}-23$ & joule/Kelvin \\
\hline Speed of Light & $c$ & 299.8 & $\mathrm{Mm} / \mathrm{sec}$. \\
\hline
\end{tabular}

Table 1: Constants used for all calculations

\begin{tabular}{|c||c|c|c|c|c|}
\hline \multicolumn{7}{|c|}{ Parameters } \\
\hline Parameter & Prob. 1 & Prob. 2 & Prob. 3 & Prob. 4 & Units \\
\hline \hline Number of Cells & 500 & 120 & 120 & 500 & N/A \\
\hline Number of Particles & 4000 & 400000 & 400000 & 10000 & N/A \\
\hline Length & 50 & 15 & 15 & 50 & Mm \\
\hline Left Albedo & 0 & 1 & 1 & 1 & N/A \\
\hline Right Albedo & 1 & 1 & 1 & 1 & N/A \\
\hline Initial Material Temp. & 0.005 & 0.005 & 0.01 & 0.01 & $\mathrm{~K}$ \\
\hline Material Density & 1.0 & 1.0 & 1.0 & 1.0 & Kg/Mm \\
\hline Number Of Time Steps & 20 & 40 & 20 & 20 & N/A \\
\hline Final Time $[\tau]$ & 1.0 & 1.0 & 1.0 & 1.0 & N/A \\
\hline Number of Groups & 1 & 1000 & N/A & 1000 & N/A \\
\hline
\end{tabular}

Table 2: Problem specif cations

Problem 1.1 is a grey purely absorbing Marshak wave benchmark problem. The face source, which is constant over time and has a magnitude of 74.925 at $\mathrm{x}=0$ and 0 elsewhere, emits photons throughout the duration of the problem $[0 \leq \tau \leq 1.0]$. The problem specif cation is included in Table 2.

Figure 1 shows the grey IMD results - "censusSolution" denotes the photon energy density as determined by the IMD method, "CensusAnalytic" represents the semi-analytic benchmark result from $\mathrm{Su}$ and Olson, "materialSolution" denotes the material energy density calculated via the IMD method and f nally "MaterialAnalytic" is the semi-analytic material energy density benchmark result from $\mathrm{Su}$ and Olson. This f gure shows that the IMD method can accurately reproduce the Su and Olson purely absorbing grey benchmark result [3]. There is a signif cant amount of noise in the low energy regions of the solution. This is related to the relatively small 


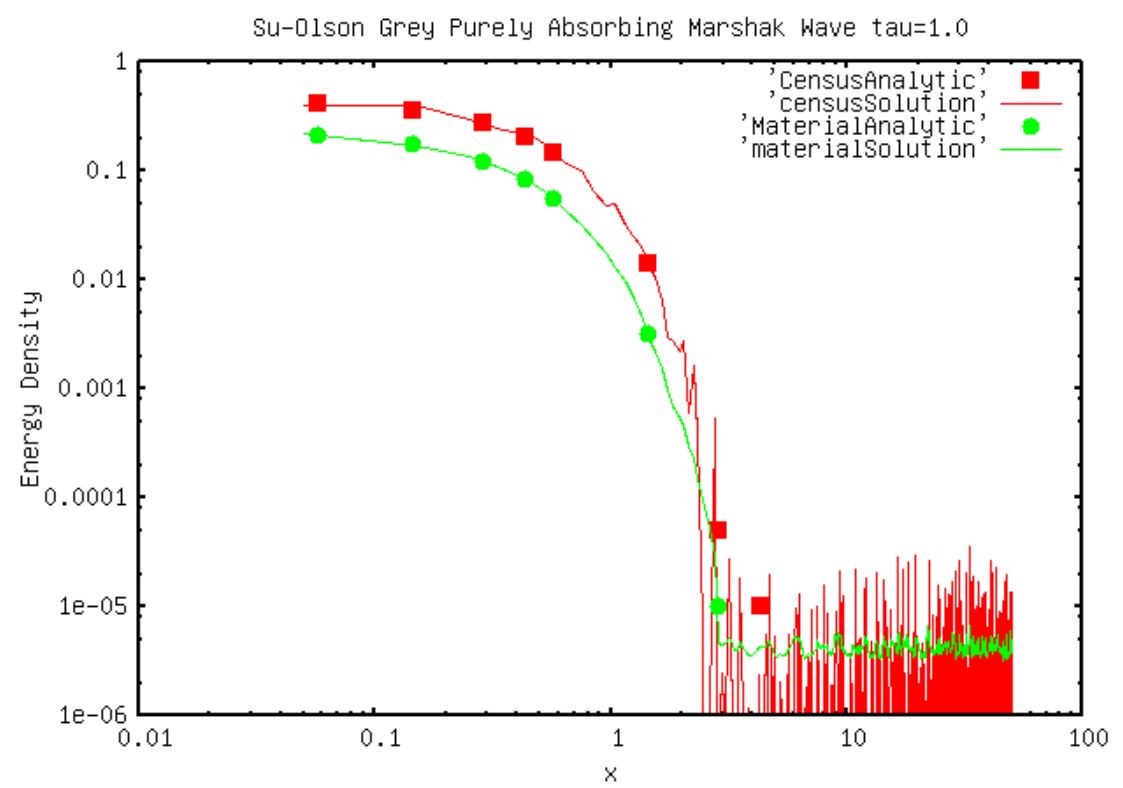

Figure 1: Grey IMD solution compared to $\mathrm{Su}$ and Olson result [3] at $\tau=1.0$. (Problem 1.1)

number of particles created in these regions because of the comparatively low contribution to the total energy of the system.

In Problem 1.2, the Su and Olson grey benchmark problem is solved using the grey IMD method with the difference formulation. For all frequency independent IMD calculations, a single opacity of 1.0 was used. The difference formulation reference temperature was chosen to be the material temperature in the associated cell. This problem specif cation is identical to that of Problem 1.1 (see Table 2).

A comparison of Figures 2 and 1 demonstrates the value of the difference formulation in reducing the variance in the solution. In Figure 2 we observe that the greatest variance reduction occurs in the region where the material is near thermal equilibrium. In fact, on the right side of the Marshak wave no particles are actually transported and the variance due to the Monte Carlo method in this region is equal to zero. The energy is simply subtracted off at the beginning of the time step during the initial differencing of the problem and is added directly back into the problem at the end of the time step.

We now consider the solution of the Su and Olson frequency-dependent benchmark problem with a picket fence opacity def ned such that: a) the bins are logarithmically spaced in frequency; b) even frequency bins have a large opacity; and c) odd frequency bins have a small opacity. The opacities are chosen such that the Planck opacity is equal to one [4]. The opacities are shown in Table 3 and the remainder of the specif cations for this problem (Problem 2.1) are listed in Table 2.

The results from the standard IMD method for Problem 2.1 (Figure 3) show that frequency dependent IMD can accurately reproduce the results of the semi-analytic picket fence opacity 
Su-0lson Grey Purely Absorbing Marshak Wave tau=1.0 with the D.F.

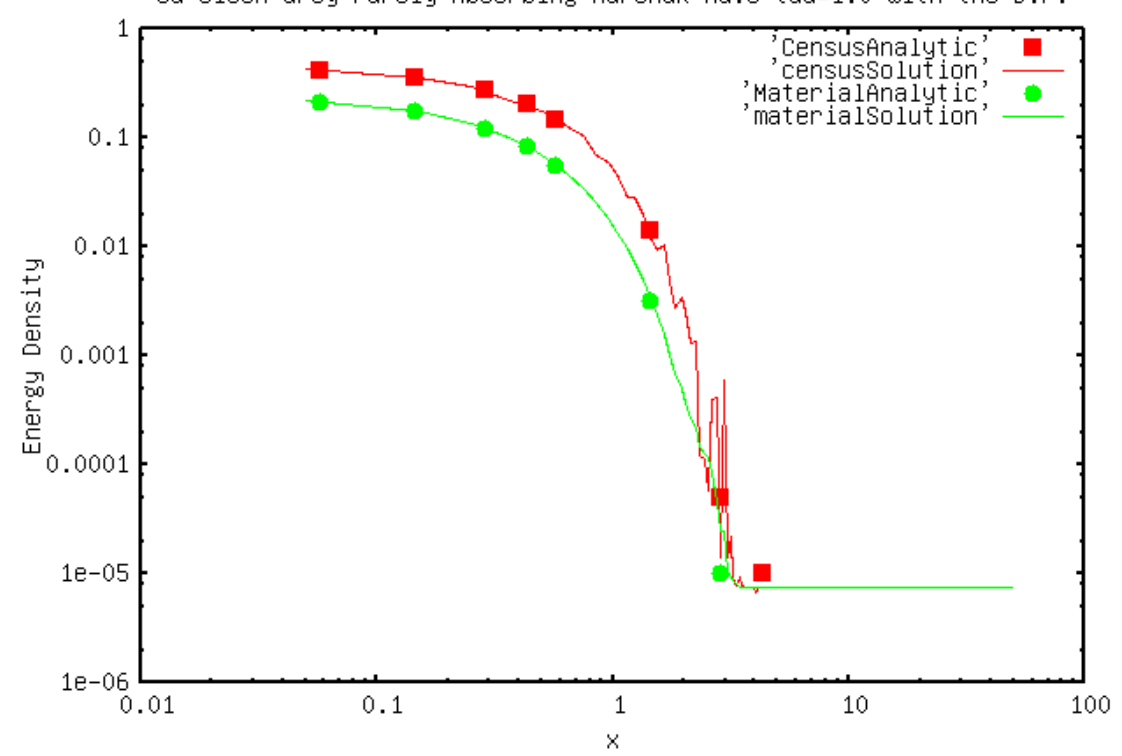

Figure 2: Grey solution with the difference formulation compared to $\mathrm{Su}$ and Olson result [3] at $\tau=1.0$. (Problem 1.2)

\begin{tabular}{|l||c|}
\hline \multicolumn{2}{|l|}{ Picket Fence Opacities } \\
\hline Small Opacity & $\frac{2}{101}$ \\
\hline Large Opacity & $\frac{200}{101}$ \\
\hline
\end{tabular}

Table 3: Opacities used to construct the opacity distribution for the frequency dependent IMD results.

benchmark def ned by Su and Olson [4]. Here "smallOpacityCensus", "largeOpacityCensus", and "materialSolution" refer to the photon energy density for the small opacity, large opacity, and the material energy density respectively, as calculated by the multigroup IMD method. The Su and Olson semi-analytic result for the small opacity photon energy density, large opacity photon energy density, and material energy density are labeled as "smallAnalytic", "largeAnalytic", and "materialAnalytic", respectively. The photon energy density associated with the large opacity is underestimated in the regions between $\mathrm{x}=2$ and the front of the Marshak wave because not enough particles are created in that region to get an accurate distribution of the thermal emission frequencies. There is signif cantly more noise in the cold region of the problem due to the low overall contribution of energy in those cells. This means that very few Monte Carlo particles register in this region resulting in overall poor statistics in these regions.

The effect of the group ref nement on the material energy density and the calculation time was also investigated (Problem 3.1). This problem has the specif cations listed in Table 2 with several choices of group structures: 1000 groups, 2000 groups, 4000 groups, 8000 groups, and 16000 groups.

Figure 4 shows the computational cost as a function of frequency group ref nement. The cost 


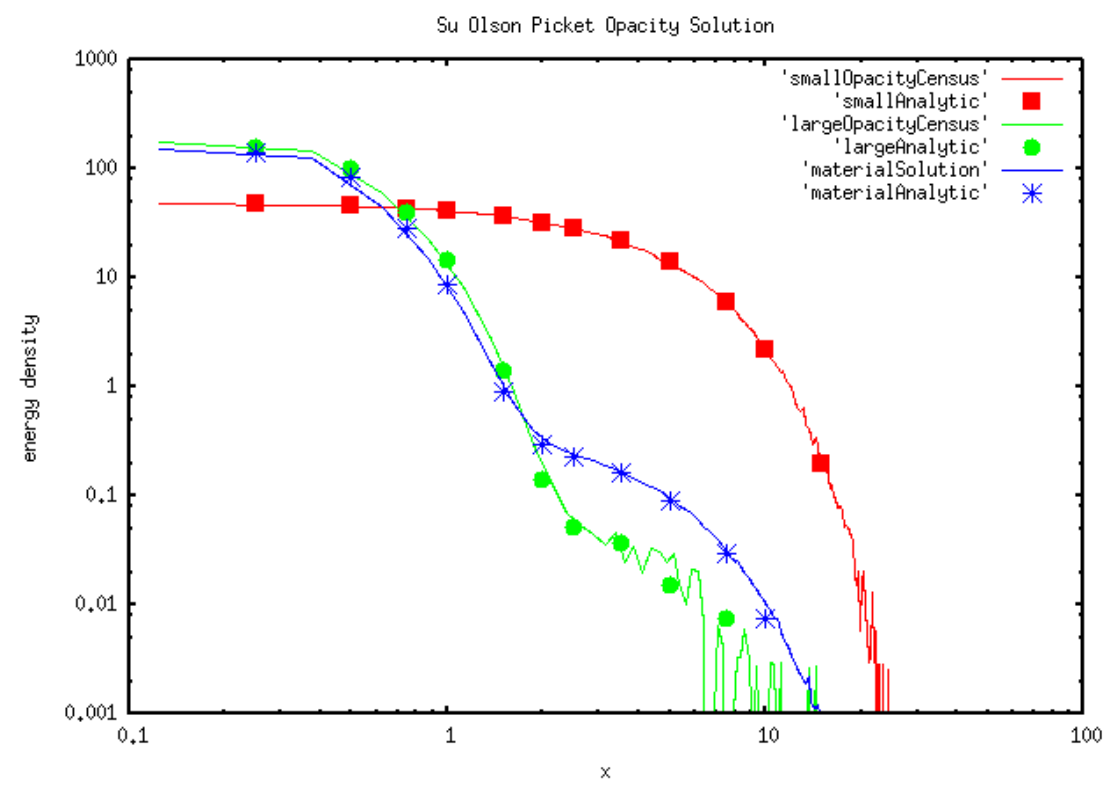

Figure 3: Normalized one dimensional frequency dependent solution compared to Su and Olson result [4] at $\tau=1.0$ with no difference formulation. (Problem 2.1)

\section{Multigroup Calculation Efficiency}

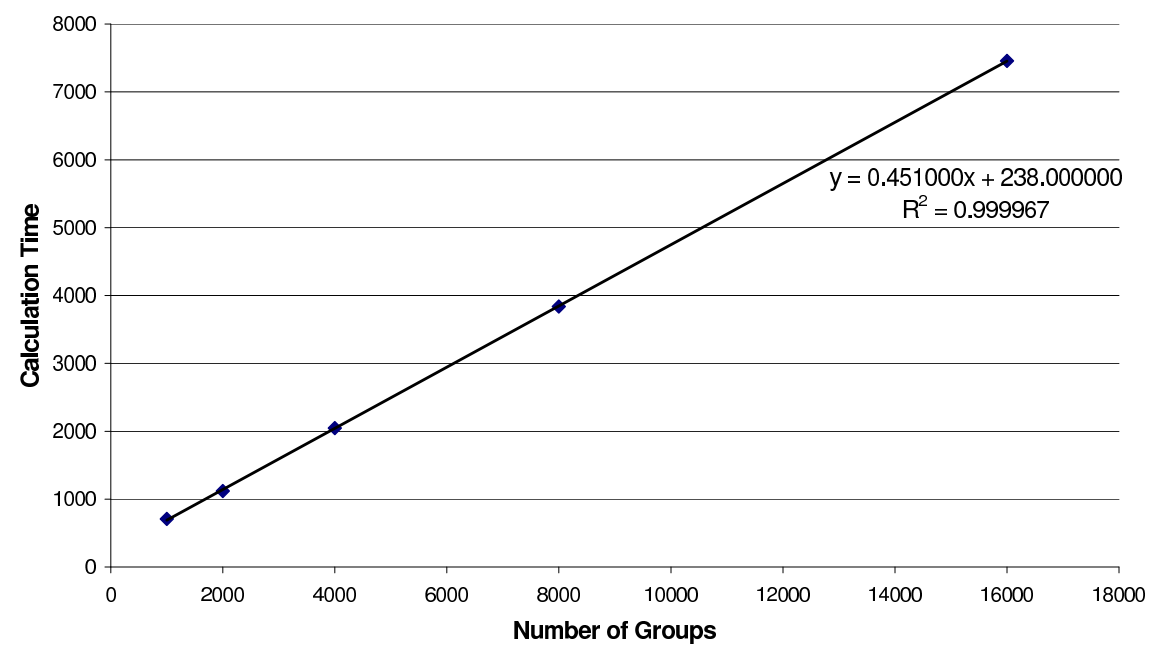

Figure 4: Computational cost as a function of number of groups used in the calculation (Problem 3.1)

of solving this multigroup problem deterministically (via a tridiagonal solver) scales linearly with 
the number of groups used. Similarly it was found that IMD scales linearly up to 16000 groups. The frequency dependence of the Monte Carlo method is determined via the random sampling from the frequency distribution functions. The cost per group curve has a slope approximatly equal to $1 / 2$ compared to a deterministic method, which would be nearly one. The $R^{2}$ value shown in Figure 4 shows how well the linear $\mathrm{ft}$ equation $y(x)$, which denotes the calculation time $(y)$ as a function of the number of groups $(x)$, matches the values that the line is being $\mathrm{ft}$ to. A value of 1 means that every value lies exactly on the linear $\mathrm{ft}$.

The next test problem explores properties of the frequency dependent implementation of IMD and the difference formulation. We generated three different solutions using the IMD method with the difference formulation and one without the difference formulation (Problem 4.1). The problem specif cations are listed in Table 2. The reference temperature distribution used in the difference formulation is a user def ned quantity. This temperature was chosen to be some fraction of the current material temperature for the associated cell. The percentages of the material temperature selected were $0.0 \%, 10.0 \%, 30.0 \%$, and $50.0 \%$. We are interested in the effect this choice has on the computational cost of the method and the degree of variance reduction.

Frequency Dependent Material Energy Density L=50

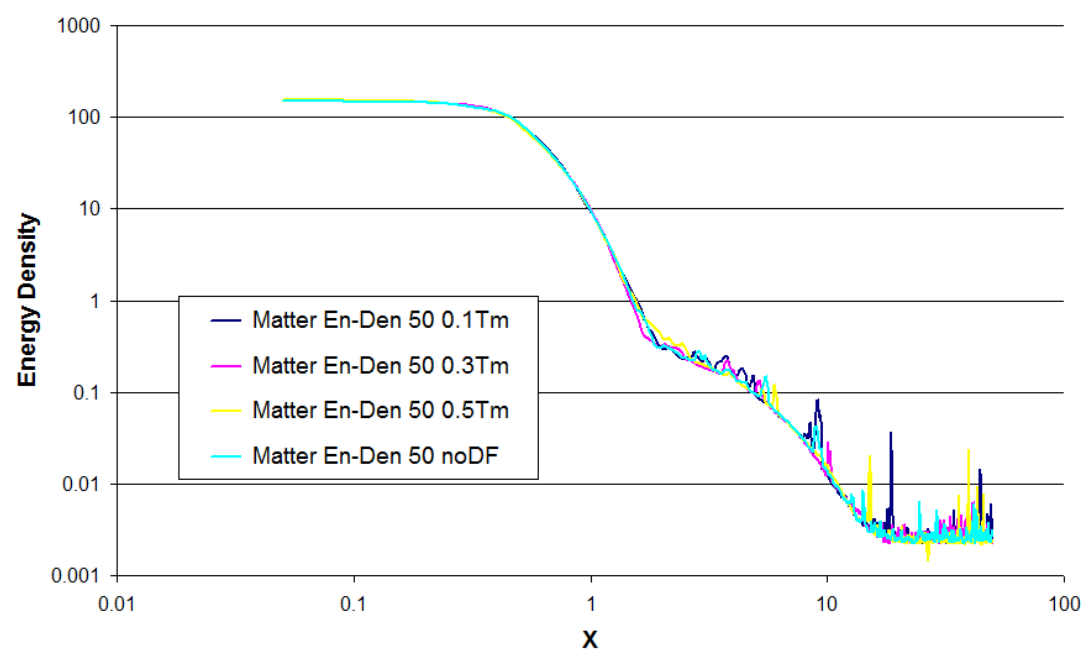

Figure 5: Non-grey result at various percentages of the material temperature for the difference formulation. (Problem 4.1)

Figure 5 shows that the IMD solutions with the difference formulation match the solution without the difference formulation. Figure 6 shows that there is not a signif cant reduction in the noise from the use of the difference formulation in the cold region of the problem. The results for the difference formulation with the reference temperature equal to that of the material temperature were not included because of numerical instability. The primary instability occurs when a cell's material energy density becomes negative as a result of negative weight particles created by the difference formulation. We discuss this further in the conclusions of this paper.

In some instances, there is also an instability in the cumulative distribution function used to 


\section{Frequency Dependent Material Energy Density L=50}

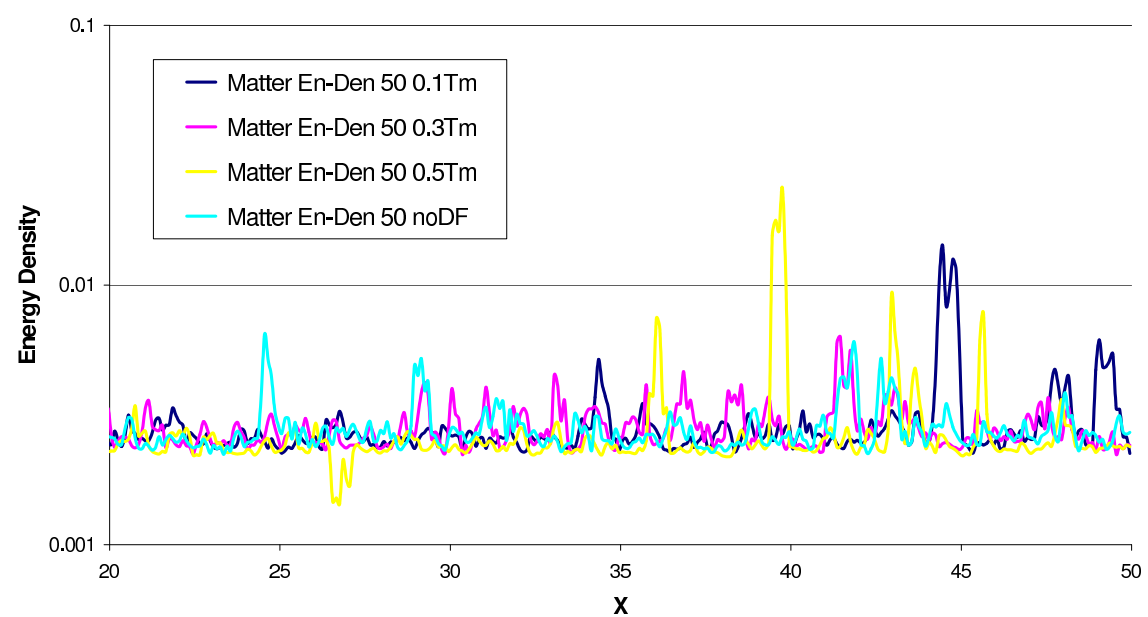

Figure 6: A magnif ed view of non-grey result at various percentages of the material temperature for the difference formulation. (Problem 4.1)

calculate the frequency distribution (equations 71 and 72) for the difference formulation source. This instability exists because of the subtraction of two nearly equal numbers when the Planckian distribution for a frequency group is low for both temperatures creating large roundoff error. This also was found to occur if the Planck integral expansion was not accurate enough. The Planckian integral expansion for a higher temperature at a given frequency can be smaller in magnitude than that of a lower temperature. This is unphysical because it is known that the Planckian at a higher temperature is larger at every frequency than the Planckian at a lower temperature.

The relative standard deviation is a measure of the statistical uncertainty of the solution compared to its mean. The relative standard deviation is simply the standard deviation of the associated cell energy density divided by the value of the energy density in that cell.

In Figure 7, "Rel STD 50 0.1Tm" denotes the relative standard deviation (Rel STD) of the material energy density with total problem length equal to $50 \mathrm{Mm}$ and the difference formulation reference temperature equal to $10 \%$ of the material temperature. The other values are def ned similarly with the exception of "Rel STD 50 noDF" which denotes the solution generated without the difference formulation. This f gure shows that with the exception of the leading edge of the Marshak wave, the relative standard deviation is consistently lower throughout the majority of the plot for all choices of the reference temperature. This is especially true for the portions of the problem that are near equilibrium (either ahead of or behind the wave front). Figure 8 shows a better view of the average standard deviation associated with the cold region of the problem. The average standard deviation is reduced as the reference temperature approaches the value of the material temperature. However, the Monte Carlo noise makes this difficult to distinguish, so the f gure of merit was calculated for each of these problems and is presented in Figure 9.

For Problem 4.1, the total f gure of merit is the sum of the f gure of merit for each cell over the whole problem. The associated relative total f gure of merit is the total f gure of merit associated with a given variance reduction technique divided by the total $f$ gure of merit without it. 


\section{Frequency Dependent Relative Standard Deviation}

(Rel. STD) of Material Energy L=50

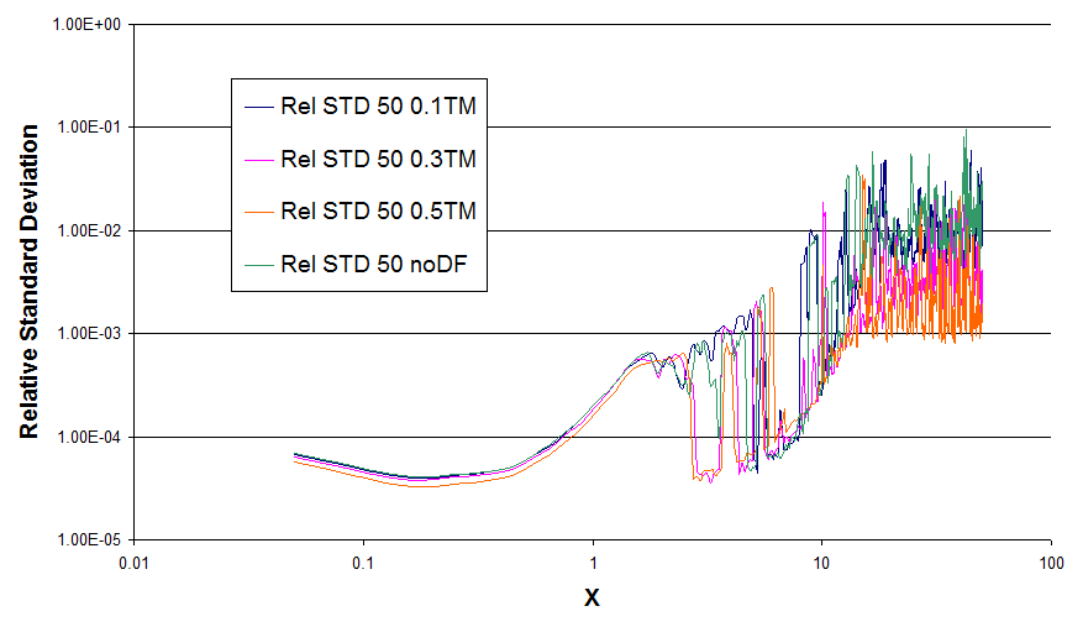

Figure 7: The relative standard deviation of the material energy density for the different realizations of Problem 4.1.

\section{Frequency Dependent Relative Standard Deviation \\ (Rel. STD) of Material Energy L=50}

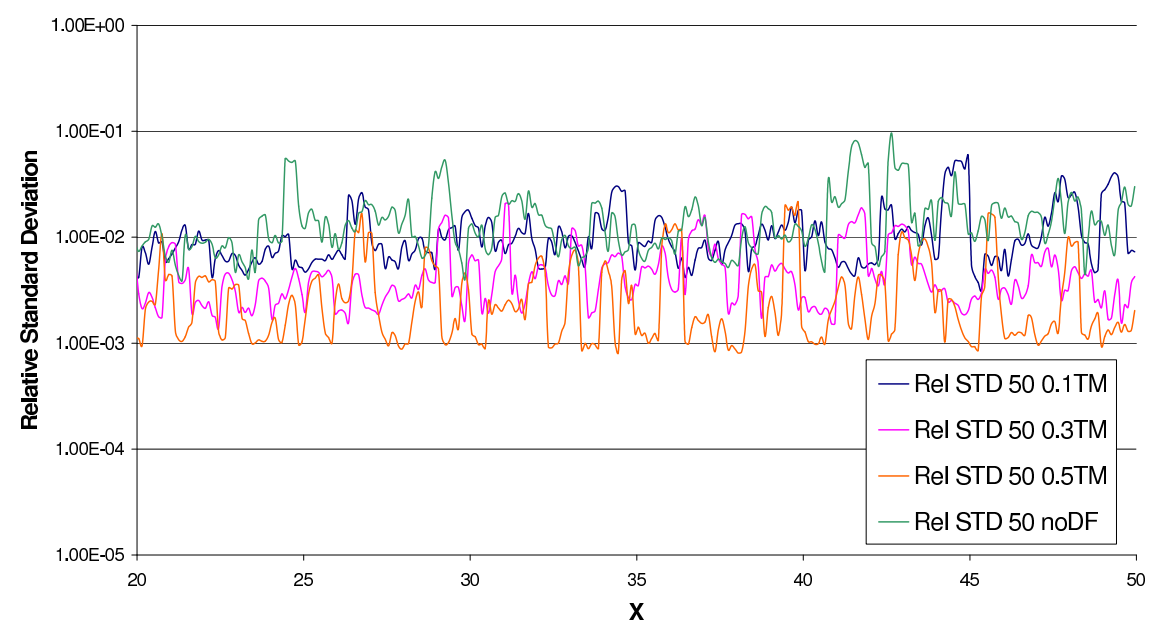

Figure 8: A zoomed in view of the relative standard deviation of the material energy density for the different realizations of Problem 4.1. 
$\%$ Increase in Total Figure of Merit

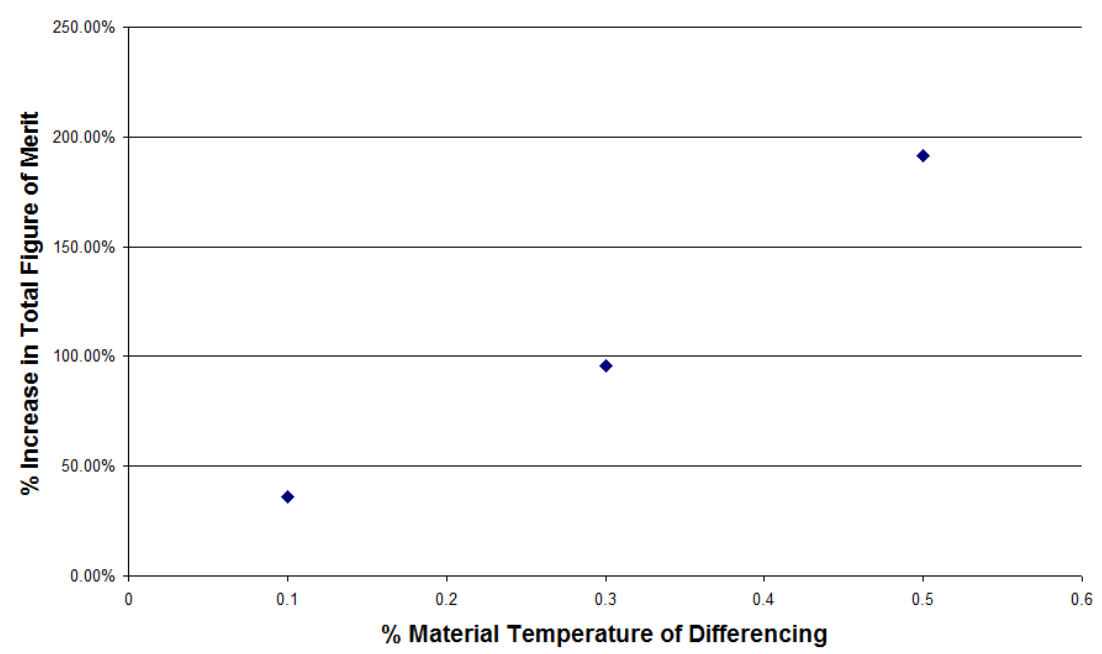

Figure 9: The relative total $f$ gure of merit for the difference formulation at various percentages of the material temperature. (Problem 4.1)

Figure 9 shows the relative increase in the f gure of merit as a function of the percent of the material temperature used for the difference formulation. This f gure shows that there is a strong increase in the f gure of merit as the difference formulation reference temperature approaches the material temperature. 


\section{Conclusions}

We have applied the difference formulation to the grey IMD method and computationally tested it. Working with a grey system is simpler than a multigroup system because there is no need to distribute the differenced energy among frequency groups. It can simply be subtracted and added to the total photon energy density. Similarly, there is no need to randomly sample from the frequency distribution of the streaming source obtained from the difference formulation. Though the grey system is a good platform for initial testing, it is not a realistic physical model of any radiative transfer applications. The multigroup method was initially successfully tested on a grey problem by setting the opacity equal to a single value for every frequency.

Using a Monte Carlo method for solving the linear system associated with a discretized multigroup diffusion equation has advantages and disadvantages. The major advantage is that there is no need to solve the matrix for each group. However, the solution of the linear system of equations using Monte Carlo can take much longer than the deterministic solution. The goal of developing IMD was to couple it with IMC. Using a Monte Carlo method to solve the diffusion equation has the advantage that it is much easier to couple to the IMC method. Using the criteria def ned by Densmore et al, it is possible to transition particles from IMD (or DDMC) to IMC [11]. For the deterministic solution, the spatial domain must be decomposed and the deterministic solution could only be coupled to the IMC solution at the end of every time step.

The reduction in variance achieved by the difference formulation, as is the case with most variance reduction techniques, comes at a cost. Without the difference formulation, the IMD Monte Carlo procedure is unconditionally stable. This is not true for the system with the difference formulation. In standard IMD, there are no negatively weighted particles created. This means that the total energy in any cell can never become less than zero. Equations 67 and 68, which represent the streaming source terms created from the difference formulation, allow the creation of negative weight particles. This means that it is possible for more negative weight than positive weight to be deposited into a single cell, causing the overall material temperature to become less than zero. One way to alleviate this is to use absorption and census suppression with a very low weight cutoff. Several test problems demonstrated how unstable the difference formulation can be even for the grey case. The stability appears to be strongly dependent on the number of particles that are used and the spatial gradient of the energy density. The difference formulation typically fails at the leading edge of the Marshak wave. It never failed in the large near-equilibrium back end of the Marshak wave or the cold portion of the material at thermal equilibrium. In fact, even if a vacuum boundary is set on the cold side of the problem, well away from the head of the Marshak wave front, the system was stable. The f gure of merit was not determined for the grey case because it would be much higher than that of the frequency dependent difference formulation. The estimation of computational efficiency of the difference formulation was limited to the frequency dependent case.

This research shows that frequency dependent IMD can be used to accurately reproduce solutions to the frequency dependent radiative transfer equations where the diffusion approximation is valid. It also shows that frequency dependent IMD is stable if the spatial and temporal discretization schemes used to generate the linear system are stable. Though this frequency dependent system is solved via IMD, frequency dependent DDMC is also possible. The primary difference between DDMC and IMD is the treatment of the temporal variable.

The difference formulation was shown to have some signif cant advantages and disadvantages when used with IMD. Implementation of the difference formulation signif cantly increases the f gure of merit in regions of the problem where the system is near equilibrium. However, there 
was no signif cant noise reduction from the frequency dependent difference formulation and it can also become unstable in regions containing sharp gradients. This is a result of the creation of large negatively weighted particles from the streaming source. These instabilities can be reduced by using a lower temperature for the difference formulation. This reduces the number of negatively weighted particles being produced and it increases the number of positively weighted particles created by the thermal emission source. These instabilities do not occur in SIMC with an implicit treatment of the reference temperature. $[9,13]$. However, comparable instabilities have been noted in SIMC when the reference temperature is treated explicitly.[13]

Several improvements to IMD could be explored, including extension of the method to two or three dimensional orthogonal meshes. However, unstructured polyhedral meshes, which are sometimes used in high energy density physics, pose difficult problems because of the possible creation of positive off diagonal elements in the coefficient matrix [1]. For the leakage probabilities to be positive, the off-diagonal elements of the coefficient matrix must all be negative.

Improved implementations of the difference formulation should also be explored. This includes automating the use of the difference formulation in different regions of the problem. The goal is to focus the use of the difference formulation where the system is near equilibrium and reduce its use in regions of sharp gradients. More work should also be performed to develop an efficient and stable algorithm for the sampling of frequency distribution function of the difference formulation streaming source. The majority of the computational work for the difference formulation is associated with the sampling of this source.

This work performed under the auspices of the U.S. Department of Energy by Lawrence Livermore National Laboratory under Contract DE-AC5207NA27344. 


\section{References}

[1] Gentile, N. A., "Implicit Monte Carlo Diffusion - An acceleration method for Monte Carlo time-dependent radiative transfer simulations", Journal of Computational Physics, 172, 543-571 (2001).

[2] Szoke, A., Brooks, E.D., "The transport equation in optically thick media", Journal of Quantitative Spectroscopy and Radiative Transfer, 91, 95-110 (2005).

[3] Su, B., Olson, G.L., "Benchmark results for the non-equilibrium Marshak diffusion problem", Journal of Quantitative Spectroscopy and Radiative Transfer, 56(3), 337-351(1996).

[4] Su, B., Olson, G.L., "Non-grey benchmark results for two temperature non-equilibrium radiative transfer", Journal of Quantitative Spectroscopy and Radiative Transfer, 62, 279-302(1999).

[5] Fleck, J. A., Canf eld, E.H., "A random walk procedure for improving the computational efficiency of the implicit Monte Carlo method for nonlinear radiation transport", Journal of Computational Physics, 54(3), 508-523 (1984).

[6] N'Kaoua, T., "Solution of the nonlinear radiative transfer equations by a fully implicit matrix Monte Carlo method coupled with the Rosseland diffusion equation via domain decomposition", SIAM Journal on Scientific and Statistical Computing, 2(3), 505-520 (1991).

[7] Clouet, J. F., Samba, G., "A hybrid symbolic Monte Carlo method for radiative transfer equations", Journal of Computational Physics, 188, 139-156 (2003)

[8] Clouet, J. F., Samba, G., "Asymptotic diffusion limit of the symbolic Monte-Carlo method for the transport equation”, Journal of Computational Physics, 195, 293-319 (2004)

[9] Brooks, E. D., McKinley, M.S., Daffin, F., Szoke, A., "Symbolic implicit Monte Carlo radiation transport in the difference formulation: a piecewise constant discretization", Journal of Computational Physics, 205, 737-754 (2005).

[10] McKinley, M. S., Brooks, E. D. III, Szoke, A., "Comparison of implicit and symbolic implicit Monte Carlo line transport with frequency weight vector extension" Journal of Computational Physics, 189, 330-349 (2003).

[11] Densmore, J. D., Urbatsch, T.J., Evans, T.M., Buksas, M.W., “A hybrid transport-diffusion method for Monte Carlo radiative-transfer simulations", Journal of Computational Physics,222, 485-503 (2007).

[12] Clevelands, M. A., "Extending the Applicability of Implicit Monte Carlo Diffusion: Frequency Dependence and Variance Reduction using the Difference Formulation", M.S. Thesis, Oregon State University, 2008.

[13] Daffin, F., McKinley, M.S., Brooks, E.D., Szoke, A., "An evaluation of the difference formulation for photon transport in a two level system", Journal of Computational Physics, 204, $27-45$ (2005).

[14] Gentile, N. A., "The difference formulation of radiation transport in implicit Monte Carlo, invited", Transactions of the American Nuclear Society, 95, 871-872 (2006).

[15] Fleck, J. A., Cummings, J.D., "An implicit Monte Carlo scheme for calculating time and frequency dependent nonlinear radiation transport", Journal of Computational Physics, 8, 313-342 (1971).

[16] Duderstadt, J. J. and Hamilton, L. J., Nuclear Reactor Analysis, John Wiley \& Sons, Inc., New York (1976).

[17] Hamerssley, J. M. and Handscomb, D. C., Monte Carlo methods, John Wiley \& Sons, Inc., New York (1964).

[18] Lewis, E. E. and Miller, Jr. D. P., Computational Methods of Neutron Transport, American Nuclear Society, Inc., Illinois (1993).

[19] Szilard, R. H., Pomraning, G.C., "Numerical transport and diffusion methods in radiative transfer", Nuclear Science and Engineering, 112, 256-269 (1992).

[20] Brooks, E. D., Szoke, A., Peterson, J.D.L., "Piecewise linear discretization of Symbolic Implicit Monte Carlo radiation transport in the difference formulation", Journal of Computational Physics, , 220, 471-497 (2006).

[21] Barnett, C., Canf eld, E., "Sampling a random variable distributed according to Planck's law", Lawrence Livermore National Laboratory, UCRL-ID-125393 (1970) (unpublished), 